\title{
Low-complexity optimization algorithm for ground network design in optical satellite networks
}

Christos Efrem, Nikolaos Lyras, Charilaos Kourogiorgas, Athanasios Panagopoulos, Pantelis-Daniel Arapoglou

Christos N. Efrem, Nikolaos K. Lyras, Charilaos I. Kourogiorgas, Athanasios D. Panagopoulos, Pantelis-Daniel Arapoglou, "Low-complexity optimization algorithm for ground network design in optical satellite networks," Proc. SPIE 11852, International Conference on Space Optics - ICSO 2020, 118525Q (11 June 2021); doi: 10.1117/12.2600010

SPIE Event: International Conference on Space Optics - ICSO 2021, 2021, Online Only 


\section{International Conference on Space Optics-ICSO 2020}

Virtual Conference

30 March-2 April 2021

Edited by Bruno Cugny, Zoran Sodnik, and Nikos Karafolas
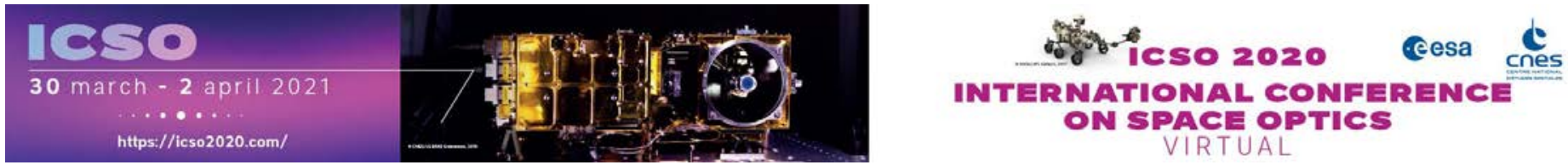

\section{Low-complexity optimization algorithm for ground network design in optical satellite networks}

\section{Cesa issopreasings denes}




\title{
Low-Complexity Optimization Algorithm for Ground Network Design in Optical Satellite Networks
}

\author{
Christos N. Efremª, Nikolaos K. Lyras ${ }^{\mathrm{a}}$, Charilaos I. Kourogiorgas ${ }^{\mathrm{a}}$, Athanasios D. Panagopoulos ${ }^{\mathrm{a}}$, \\ Pantelis-Daniel Arapoglou ${ }^{\mathrm{b}}$

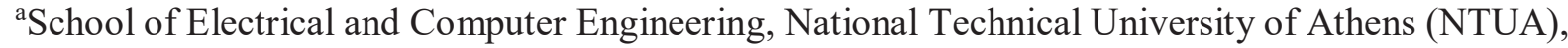 \\ 15780, Zografou, Athens, Greece \\ bEuropean Space Agency, ESTEC-ESA, 2200 AG Noordwijk, The Netherlands \\ e-mail: chefr@central.ntua.gr; lyrasnikos@central.ntua.gr; harkour@mail.ntua.gr; \\ thpanag@ece.ntua.gr; pantelis-daniel.arapoglou@esa.int
}

\begin{abstract}
Free space optics (FSO) is considered a promising technology for satellite communications due to its various advantages over radio-frequency (RF) systems, such as higher throughput, lower energy consumption and smaller mass. Nevertheless, optical satellite communication systems are heavily affected by atmospheric impairments, mainly by clouds. In order to cope with cloud coverage, site diversity technique is employed at the expense of installing extra optical ground stations (OGSs). As a consequence, the interest in ground network optimization is rapidly increasing with the aim to guarantee a given service availability. In this paper, a low-complexity optimization algorithm for ground network design in optical geostationary (GEO) satellite systems is presented, taking into account the spatial correlation between sites. Specifically, the objective is to choose a group of candidate OGSs that minimizes the overall cost of the ground network and meets certain availability requirements for every time period (thus incorporating the temporal variability of cloud coverage). Moreover, an extension of the methodology to optical medium-Earth-orbit (MEO) satellite systems is provided. Lastly, the performance of the proposed algorithm is evaluated via numerical experiments.
\end{abstract}

Keywords: Optical satellite communications, site diversity, cloud-free line-of-sight (CFLOS) probability, system availability, ground station selection, optimization.

\section{INTRODUCTION}

Recently, there has been a lot of interest in the utilization of free space optics (FSO) in satellite communication networks. In particular, FSO technology is able to provide larger bandwidth, higher data-rates as well as increased security [1]. Several atmospheric phenomena affect the propagation of optical signals, but the cloud coverage is the major fading mechanism that results in link outage. As a result, the optical link can be modeled as an on/off channel depending on whether or not there is a cloud blockage [2].

Site diversity is an effective technique which can be employed to mitigate the cloud coverage, combining the optical signals transmitted by geographically distributed OGSs towards the same satellite [1]-[4]. In spite of the fact that the OGSs are preferably selected to have large separation distances (thus very low spatial correlation), this is not always the case in practice, so their spatial correlation should be taken into account when estimating the system availability. To this end, the cloud-free line-of-sight (CFLOS) probability, for either a single or multiple links, has been studied extensively in the literature [2]-[3], [5]-[8].

Furthermore, the optimum selection of OGSs has become an interesting research topic nowadays. For example, GEO satellite systems have been examined in [7]-[12], MEO satellite networks have been analyzed in [13]-[15], and low-Earthorbit (LEO) satellite systems have been studied in [16]. All these approaches provide useful tools and optimization algorithms in order to design the ground segment of optical satellite networks.

The main contribution of this paper is the presentation of a unified methodology for the optimum selection of OGSs in optical GEO/MEO satellite networks in order to minimize the overall cost of the ground network and satisfy given 
availability requirements per time period. To the best of our knowledge, this is the first work that studies the minimization of the total cost of OGSs (not merely their number) and, at the same time, takes into consideration not only the temporal variability of clouds, but also the spatial correlation of OGSs.

The rest of the paper is organized as follows. In Section 2, the optimization problem is formulated, while a low-complexity optimization algorithm is presented in Section 3. In addition, an extension of the proposed methodology to optical MEO satellite systems is described in Section 4 and simulation experiments are provided in Section 5. Lastly, some conclusions and directions for future work are given in Section 6.

\section{PROBLEM STATEMENT}

To begin with, we consider an optical GEO satellite network with $\mathcal{K}=\{1,2, \ldots, K\}$ being the set of candidate OGSs and $\mathcal{T}=\{1,2, \ldots, T\}$ being the set of time periods (e.g., months). Subsequently, we attempt to find a subset $\mathcal{I}$ of $\mathcal{K}$ that minimizes the overall cost of the ground network, $C(\mathcal{I})$, under availability constraints for each time period. As a result, we can formulate the following optimization problem:

$$
\begin{array}{cl}
\underset{\mathcal{I}}{\operatorname{minimize}} & C(\mathcal{I})=\sum_{i \in \mathcal{I}} c_{i} \\
\text { subject to } & A_{\tau}(\mathcal{I}) \geq A_{\tau}^{\text {th }}, \quad \forall \tau \in \mathcal{T} \\
& \mathcal{I} \subseteq \mathcal{K}
\end{array}
$$

where $c_{i}>0$ is the cost of OGS $i, A_{\tau}(\mathcal{I})$ is the system availability (or, the joint CFLOS probability) in time period $\tau$ achieved by the set $\mathcal{I}$ of OGSs, and $A_{\tau}^{\text {th }}$ is the system availability threshold for time period $\tau$. More precisely, the system availability $A_{\tau}(\mathcal{I})$, i.e., the probability of having at least one OGSs in $\mathcal{I}$ available in time period $\tau$, is expressed as follows:

$$
\begin{gathered}
A_{\tau}(\mathcal{I})=\mathbb{P}\left(\bigcup_{i \in \mathcal{I}}\left\{X_{i}<x_{i, \tau}^{\mathrm{th}}\right\}\right)=1-\mathbb{P}\left(\bigcap_{i \in \mathcal{I}}\left\{X_{i} \geq x_{i, \tau}^{\mathrm{th}}\right\}\right)=1-\int_{\mathcal{D}_{\mathcal{I}, \tau}} \varphi_{\mathbf{x}_{\mathcal{I}}}\left(\boldsymbol{x}_{\mathcal{I}}\right) d \boldsymbol{x}_{\mathcal{I}} \\
\varphi_{\mathbf{x}_{\mathcal{I}}}\left(\boldsymbol{x}_{\mathcal{I}}\right)=\frac{\exp \left(-0.5 \boldsymbol{x}_{\mathcal{I}}^{\top} \mathbf{R}_{\mathcal{I}}^{-1} \boldsymbol{x}_{\mathcal{I}}\right)}{\sqrt{(2 \pi)^{|\mathcal{I}|} \operatorname{det}\left(\mathbf{R}_{\mathcal{I}}\right)}}
\end{gathered}
$$

where $\mathbf{X}_{\mathcal{I}}=\left[X_{i}\right]_{i \in \mathcal{I}}^{\top}$ is a random vector of zero-mean unit-variance normally distributed random variables and $x_{i, \tau}^{\text {th }}=Q^{-1}\left(1-P_{i, \tau}\right)$ with $P_{i, \tau}$ being the individual CFLOS probability of OGS $i$ in time period $\tau$; this probability depends on the altitude and the elevation angle of the OGS and can be estimated by following the methodology presented in [5] using the integrated-liquid-water-content (ILWC) statistics [2]. Also, $\mathcal{D}_{\mathcal{I}, \tau}=\left\{\boldsymbol{x}_{\mathcal{I}}=\left[x_{i}\right]_{i \in \mathcal{I}}^{\top} \in \mathbb{R}^{|\mathcal{I}|} \mid x_{i} \geq x_{i, \tau}^{\text {th }}, \forall i \in \mathcal{I}\right\}$ is the domain of integration, $\varphi(\cdot)$ is the probability density function (PDF) of the multivariate normal distribution, $d \boldsymbol{x}_{\mathcal{I}}=\prod_{i \in \mathcal{I}} d x_{i}$ and $\mathbf{R}_{\mathcal{I}}=\left[\rho_{i, j}\right]_{i, j \in \mathcal{I}}$ is the correlation matrix (symmetric and positive-definite) that captures the spatial correlation of OGSs and whose entries are given by [17]: 


$$
\rho_{i, j}=0.35 \exp \left(-\frac{d_{i, j}}{7.8}\right)+0.65 \exp \left(-\frac{d_{i, j}}{225.3}\right)
$$

where $d_{i, j}$ is the distance between OGSs $i$ and $j$, which is expressed in km; observe that $d_{i, i}=0 \Rightarrow \rho_{i, i}=1$. Last but not least, the multivariate normal integral in (2) can be calculated using readily available functions from scientific programming software, such as Matlab, based on quasi-Monte Carlo methods [18].

\section{OPTIMIZATION ALGORITHM}

A naive procedure to globally solve problem (1) is the exhaustive-enumeration algorithm which checks all the $2^{K}$ subsets of $\mathcal{K}$. Nevertheless, such an algorithm has exponential complexity in the number of candidate OGSs. To this end, we develop an efficient optimization algorithm that is able to find a near-optimal solution with much lower complexity. In the following analysis, $\mathcal{S}$ denotes the set of OGSs selected so far by the algorithm; at the beginning of the algorithm $\mathcal{S}$ is empty.

Firstly, we define a penalty function which accounts for the total violation of the availability constraints when OGS $i$ is added to $\mathcal{S}$ :

$$
\vartheta_{\mathcal{S}}(i)=\sum_{\tau \in \mathcal{T}} \max \left(A_{\tau}^{\text {th }}-A_{\tau}(\mathcal{S} \cup\{i\}), 0\right), \quad \forall i \in \mathcal{K} \backslash \mathcal{S}
$$

In addition, we define another function as the product of the cost $c_{i}$ and the penalty function $\vartheta_{\mathcal{S}}(i)$ :

$$
\sigma_{\mathcal{S}}(i)=c_{i} \cdot \vartheta_{\mathcal{S}}(i), \quad \forall i \in \mathcal{K} \backslash \mathcal{S}
$$

In essence, the last function encapsulates the increase in cost together with the overall constraint-violation yielded by selecting OGS $i$. The pseudocode of the proposed algorithm is given in Table 1. In every iteration, the OGS with the minimum $\sigma_{\mathcal{S}}(i)$ is chosen from the remaining OGSs and then added to $\mathcal{S}$. This process is repeated until satisfying all the availability constraints, since $\sigma_{\mathcal{S}}(i)=0 \Leftrightarrow \vartheta_{\mathcal{S}}(i)=0 \Leftrightarrow A_{\tau}(\mathcal{S} \cup\{i\}) \geq A_{\tau}^{\text {th }}, \forall \tau \in \mathcal{T}$.

Last but not least, the maximum number of subsets checked by the algorithm is $\sum_{m=0}^{K-1}(K-m)=\sum_{m=1}^{K} m=\frac{K(K+1)}{2}=$ $=O\left(K^{2}\right) \ll 2^{K}$, which is polynomial (quadratic) in the number of candidate OGSs.

\section{EXTENSION TO OPTICAL MEO SATELLITE SYSTEMS}

Afterwards, an extension of the proposed approach to optical satellite networks with a single MEO satellite is presented. First of all, we introduce another set $\mathcal{B}=\{1,2, \ldots, B\}$ which represents the MEO-satellite orbital positions, i.e., we perform a discretization of the orbit of the MEO satellite. Secondly, we should make the following modifications:

- The new availability constraints, per time period and orbital position, are: $A_{\tau, b}(\mathcal{I}) \geq A_{\tau, b}^{\text {th }}, \quad \forall \tau \in \mathcal{T}, \forall b \in \mathcal{B}$.

- The quantities $A_{\tau}(\mathcal{I}), A_{\tau}^{\text {th }}, x_{i, \tau}^{\text {th }}, P_{i, \tau}$ and $\mathcal{D}_{\mathcal{I}, \tau}$ are replaced by $A_{\tau, b}(\mathcal{I}), A_{\tau, b}^{\text {th }}, x_{i, \tau, b}^{\text {th }}, P_{i, \tau, b}$ and $\mathcal{D}_{\mathcal{I}, \tau, b}$, respectively. In other words, we replace every subscript $\tau$ with $\tau, b$. 
- The penalty function becomes $\vartheta_{\mathcal{S}}(i)=\sum_{\tau \in \mathcal{T}} \sum_{b \in \mathcal{B}} \max \left(A_{\tau, b}^{\text {th }}-A_{\tau, b}(\mathcal{S} \cup\{i\}), 0\right), \quad \forall i \in \mathcal{K} \backslash \mathcal{S}$.

Now, the algorithm presented in Table 1 can be used exactly as it is. Finally, with regard to optical satellite networks with multiple MEO satellites (i.e., full MEO constellation), the desired system availability can be achieved if every selected OGS is equipped with adequate number of terminals [15].

Table 1. Optimization Algorithm for OGS selection in optical satellite networks.

1: $\mathcal{S}:=\varnothing$
2: repeat
3: $\quad i^{*}:=\arg \min \left\{\sigma_{\mathcal{S}}(i) \mid i \in \mathcal{K} \backslash \mathcal{S}\right\}$
4: $\quad \sigma^{*}:=\sigma_{\mathcal{S}}\left(i^{*}\right)$
5: $\quad \mathcal{S}:=\mathcal{S} \cup\left\{i^{*}\right\}$
6: until $\sigma^{*}=0$
7: return $\mathcal{S}$

\section{SIMULATION EXPERIMENTS}

In this section, the performance of the proposed algorithm is investigated via numerical simulations, assuming an optical GEO satellite system with $K=15$ candidate OGSs (shown in Table 2), $T=12$ time periods (the months of a year), and $A_{\tau}^{\text {th }}=A^{\text {th }}, \forall \tau \in \mathcal{T}$ (in what follows, $A^{\text {th }}$ is referred to as the system availability threshold). In addition, all results have been averaged over 100 independent and identically distributed (i.i.d.) scenarios, where the OGS costs $c_{i} \sim$ uniform $\{4,5,6,7,8\}$. Moreover, the relative error is used as a performance indicator, which is defined by:

$$
\varepsilon_{\mathrm{rel}}=\frac{C(\mathcal{S})-C^{*}}{C^{*}}
$$

where $C(\mathcal{S})$ is the overall cost achieved by the proposed algorithm and $C^{*}$ is the optimum cost obtained from the exhaustive-enumeration algorithm. Obviously, $C(\mathcal{S}) \geq C^{*}$.

The average values of $C(\mathcal{S}), C^{*}$ and $\varepsilon_{\text {rel }}$ versus the system availability threshold are shown in Table 3 . In particular, the optimum cost $C^{*}$ increases with $A^{\text {th }}$; this is expected, since greater availability requirement results in higher cost for the ground network development. Last but not least, the achieved relative error ranges from 6.8 to $17.6 \%$. 
Table 2. Candidate OGS locations.

\begin{tabular}{|c|c|c|c|c|c|}
\hline No. & Site & $\begin{array}{c}\text { Latitude } \\
{\left[{ }^{\mathbf{0}} \mathbf{N}\right]}\end{array}$ & $\begin{array}{c}\text { Longitude } \\
{\left[{ }^{\mathbf{0}} \mathbf{E}\right]}\end{array}$ & $\begin{array}{c}\text { Altitude } \\
{[\mathbf{k m}]}\end{array}$ & $\begin{array}{c}\text { Elevation Angle } \\
{\left[{ }^{0}\right]}\end{array}$ \\
\hline 1 & Malargue & -35.48 & -69.59 & 1.4 & 48.1 \\
\hline 2 & Vernon & 34.21 & -99.4 & 0.4 & 43.6 \\
\hline 3 & Manassas & 38.78 & -77.57 & 0.12 & 45.1 \\
\hline 4 & Steele Valley & 33.76 & -117.3 & 0.6 & 43.9 \\
\hline 5 & Washington & 39.9 & -77.04 & 0.2 & 43.4 \\
\hline 6 & Oklahoma & 35.44 & -97.53 & 0.45 & 30.5 \\
\hline 7 & Santiago & -33.43 & -70.64 & 0.3 & 30.4 \\
\hline 8 & South Mountain (CA) & 34.33 & -118.99 & 0.4 & 45.2 \\
\hline 9 & Las Vegas & 36.12 & -115.2 & 0.65 & 76 \\
\hline 10 & Buenos Aires & -34.6 & -58.41 & 0.3 & 46.3 \\
\hline 11 & Lima & -11.94 & -76.72 & 0.8 & 46.3 \\
\hline 12 & Sao Paulo & -23.55 & -46.65 & 0.7 & 75.6 \\
\hline 13 & Dallas & 32.74 & -96.9 & 0.16 & 44.4 \\
\hline 14 & Lurin & -12.28 & -76.85 & 0.1 & 0.22 \\
\hline 15 & Maryland & 39.38 & -77.08 & & 32 \\
\hline
\end{tabular}

Table 3. Performance evaluation of the proposed algorithm.

\begin{tabular}{|c|c|c|c|}
\hline$A^{\text {th }}(\%)$ & $C(\mathcal{S})$ & $C^{*}$ & $\varepsilon_{\text {rel }}(\%)$ \\
\hline 98.1 & 22.52 & 19.17 & 17.6 \\
\hline 98.4 & 22.55 & 19.68 & 14.7 \\
\hline 98.7 & 22.39 & 20.18 & 11.0 \\
\hline 99.0 & 26.63 & 22.94 & 16.2 \\
\hline 99.3 & 27.58 & 24.47 & 12.8 \\
\hline 99.6 & 32.18 & 28.62 & 12.5 \\
\hline 99.9 & 40.54 & 37.92 & 6.8 \\
\hline
\end{tabular}

\section{CONCLUSION \& FUTURE WORK}

In summary, the optimum selection of OGSs in optical GEO/MEO satellite networks has been studied, in order to minimize the overall cost of the ground network and satisfy given availability requirements for each time period. The proposed approach captures the spatial correlation between the OGSs as well as the temporal variability of clouds. An efficient optimization algorithm has also been developed to find near-optimal solutions with affordable complexity. Finally, according to the simulation experiments, the proposed algorithm achieves an average relative error in the order of $10-15 \%$. Although this algorithm has much lower complexity than the exhaustive enumeration of all subsets/combinations, it is a short-sighted heuristic algorithm that performs a greedy selection of the next OGS in each iteration. Therefore, it would be particularly useful to design more sophisticated optimization algorithms with better performance. 


\section{REFERENCES}

[1] H. Kaushal and G. Kaddoum, "Optical Communication in Space: Challenges and Mitigation Techniques," IEEE Communications Surveys \& Tutorials, vol. 19, no. 1, pp. 57-96, First Quarter 2017.

[2] N. K. Lyras, C. I. Kourogiorgas and A. D. Panagopoulos, "Cloud Attenuation Statistics Prediction From Ka-Band to Optical Frequencies: Integrated Liquid Water Content Field Synthesizer," IEEE Transactions on Antennas and Propagation, vol. 65, no. 1, pp. 319-328, Jan. 2017.

[3] C. I. Kourogiorgas et al., "Capacity Statistics Evaluation for Next Generation Broadband MEO Satellite Systems," IEEE Transactions on Aerospace and Electronic Systems, vol. 53, no. 5, pp. 2344-2358, Oct. 2017.

[4] A. D. Panagopoulos, P. M. Arapoglou and P. G. Cottis, "Satellite communications at Ku, Ka, and V bands: Propagation impairments and mitigation techniques," IEEE Communications Surveys \& Tutorials, vol. 6, no. 3, pp. 2-14, Third Quarter 2004.

[5] N. K. Lyras, C. I. Kourogiorgas and A. D. Panagopoulos, "Cloud Free Line of Sight Prediction Modeling for Optical Satellite Communication Networks," IEEE Communications Letters, vol. 21, no. 7, pp. 1537-1540, July 2017.

[6] M. S. Net, I. D. Portillo, E. Crawley and B. Cameron, "Approximation methods for estimating the availability of optical ground networks," IEEE/OSA Journal of Optical Communications and Networking, vol. 8, no. 10, pp. 800-812, Oct. 2016.

[7] C. Fuchs and F. Moll, "Ground station network optimization for space-to-ground optical communication links," IEEE/OSA Journal of Optical Communications and Networking, vol. 7, no. 12, pp. 1148-1159, Dec. 2015.

[8] S. Poulenard, M. Crosnier and A. Rissons, "Ground segment design for broadband geostationary satellite with optical feeder link," IEEE/OSA Journal of Optical Communications and Networking, vol. 7, no. 4, pp. 325-336, April 2015.

[9] N. K. Lyras, C. N. Efrem, C. I. Kourogiorgas and A. D. Panagopoulos, "Optimum Monthly Based Selection of Ground Stations for Optical Satellite Networks," IEEE Communications Letters, vol. 22, no. 6, pp. 1192-1195, June 2018.

[10] S. Gong et al., "Network Availability Maximization for Free-Space Optical Satellite Communications," IEEE Wireless Communications Letters, vol. 9, no. 3, pp. 411-415, March 2020.

[11]C. N. Efrem and A. D. Panagopoulos, "Globally Optimal Selection of Ground Stations in Satellite Systems with Site Diversity," IEEE Wireless Communications Letters, vol. 9, no. 7, pp. 1101-1104, July 2020.

[12] C. N. Efrem and A. D. Panagopoulos, "Minimizing the Installation Cost of Ground Stations in Satellite Networks: Complexity, Dynamic Programming and Approximation Algorithm," IEEE Wireless Communications Letters, doi: 10.1109/LWC.2020.3031717.

[13] N. K. Lyras, C. N. Efrem, C. I. Kourogiorgas, and A. D. Panagopoulos, "Medium earth orbit optical satellite communication networks: Ground terminals selection optimization based on the cloud-free line-of-sight statistics," International Journal of Satellite Communications and Networking, vol. 37, no. 4, pp. 370-384, July/August 2019.

[14]C. N. Efrem, N. K. Lyras, C. I. Kourogiorgas, A. D. Panagopoulos and P. Arapoglou, "Cloud Free LOS Probability Estimation for MEO Optical Satellite Systems and Optical Satellite Network Dimensioning," 2020 14th European Conference on Antennas and Propagation (EuCAP), Copenhagen, Denmark, 2020, pp. 1-5.

[15] N. K. Lyras, C. N. Efrem, C. I. Kourogiorgas, A. D. Panagopoulos and P.-D. Arapoglou, "Optimizing the Ground Network of Optical MEO Satellite Communication Systems," IEEE Systems Journal, vol. 14, no. 3, pp. 39683976, Sept. 2020.

[16] I. del Portillo, M. Sanchez, B. Cameron and E. Crawley, "Optimal location of optical ground stations to serve LEO spacecraft," 2017 IEEE Aerospace Conference, Big Sky, MT, 2017, pp. 1-16.

[17]L. Luini and C. Capsoni, "Modeling High-Resolution 3-D Cloud Fields for Earth-Space Communication Systems," IEEE Transactions on Antennas and Propagation, vol. 62, no. 10, pp. 5190-5199, Oct. 2014.

[18] Genz, A., and F. Bretz, "Comparison of Methods for the Computation of Multivariate t Probabilities," Journal of Computational and Graphical Statistics, vol. 11, no. 4, 2002, pp. 950-971. 